\title{
Coronal loop seismology using multiple transverse loop oscillation harmonics
}

\author{
T. Van Doorsselaere, V. M. Nakariakov, and E. Verwichte
}

\author{
Centre for Fusion, Space, and Astrophysics, Department of Physics, University of Warwick, Coventry, CV4 7AL, UK \\ e-mail: t.van-doorsselaere@warwick .ac.uk
}

Received 3 May 2007 / Accepted 2 August 2007

\section{ABSTRACT}

\begin{abstract}
Context. TRACE observations (23/11/1998 06:35:57-06:48:43 UT) in the $171 \AA$ Aandpass of an active region are studied. Coronal loop oscillations are observed after a violent disruption of the equilibrium.

Aims. The oscillation properties are studied to give seismological estimates of physical quantities, such as the density scale height. Methods. A loop segment is traced during the oscillation, and the resulting time series is analysed for periodicities.

Results. In the loop segment displacement, two periods are found: $435.6 \pm 4.5 \mathrm{~s}$ and $242.7 \pm 6.4 \mathrm{~s}$, consistent with the periods of the fundamental and 2 nd harmonic fast kink oscillation. The small uncertainties allow us to estimate the density scale height in the loop to be $109 \mathrm{Mm}$, which is about double the estimated hydrostatical value of $50 \mathrm{Mm}$.

Because a loop segment is traced, the amplitude dependence along the loop is found for each of these oscillations. The obtained spatial information is used as a seismological tool to give details about the geometry of the observed loop.
\end{abstract}

Key words. Sun: corona - Sun: magnetic fields - Sun: oscillations - Sun: UV radiation

\section{Introduction}

In the last decade, a wealth of oscillatory phenomena have been discovered in the solar corona (for an overview, see Nakariakov $\&$ Verwichte 2005). These oscillatory phenomena are a tool to do MHD coronal seismology with (Roberts et al. 1984).

Of particular interest are transverse coronal loop oscillations, i.e. rapidly damped oscillations of coronal loops displacing the loop axis. In recent years, they have received a lot of attention from observers and modellers alike. The oscillations are believed to be fast magnetosonic kink modes, but the mechanism responsible for their excitation and the rapid damping is still under debate.

These oscillatory events were first observed by Aschwanden et al. (1999); Nakariakov et al. (1999). Later on, oscillations in a sample of 17 loops were studied and analysed by Schrijver et al. (2002); Aschwanden et al. (2002). Recently, Verwichte et al. (2004) found two events with signatures of 2 different periodicities in a single loop in an arcade. Wang \& Solanki (2004) found oscillations with a vertical polarisation, and Li \& Gan (2006) observed oscillations in shrinking loops.

Fast magnetosonic kink oscillations were first studied analytically by Zaitsev \& Stepanov (1975). The dispersion relation for these modes was later independently derived by Edwin \& Roberts (1983). To explain the damping, several theories exist: resonant absorption, wave leakage, phase mixing,...

Resonant absorption as a damping mechanism has received the most attention: in this mechanism a resonance is set up where the local Alfvén frequency matches the global oscillation frequency. In this resonance, energy is converted from the global mode to local Alfvén modes. Early analytical work on this mechanism was done by Goossens et al. (1995); Ruderman \& Roberts (2002) and numerical modelling was done by Van Doorsselaere et al. (2004a) to extend the standard loop model to radially inhomogeneous loops. Loop curvature was added by Van Doorsselaere et al. (2004b); Terradas et al. (2006), and longitudinal density stratification was added by Andries et al. (2005b).

Resonant absorption provides an efficient mechanism to convert global oscillations into localized Alfvén modes. However, observational signatures of the generated Alfvén modes, which would confirm this hypothesis, are difficult to measure.

A statistical study was performed by Ofman \& Aschwanden (2002) in an attempt to pin down the damping mechanism. They concluded that the oscillations are most likely damped by phase mixing with anomalously high shear viscosity. It has not been demonstrated how phase mixing as a damping mechanism of waves in coronal loops as coherent structures would work. Also, it has yet to be demonstrated how the mechanism would operate in multi-stranded loop.

In the statistical study, however, some damping mechanisms were treated improperly, e.g. resonant absorption by assuming that the length and width of the loop are proportional to the inhomogeneity scale, (Goossens et al. 2002) and the large error bars on the observations do not currently allow to distinguish between several damping mechanisms. A larger sample size and more precise observations are needed to discriminate between damping mechanisms.

Because of inherent problems with the observational study of the solar corona (line-of-sight integration and the high density contrast with the photosphere), it is practically impossible to measure the coronal density and the coronal magnetic field. Some attempts to measure the density were undertaken (Aschwanden et al. 2003) and the average value of the magnetic field was estimated by Lin et al. $(2000,2004)$, but the error bars on the results are large.

It is, however, possible to measure the density and magnetic field by doing coronal seismology (Uchida 1970; 

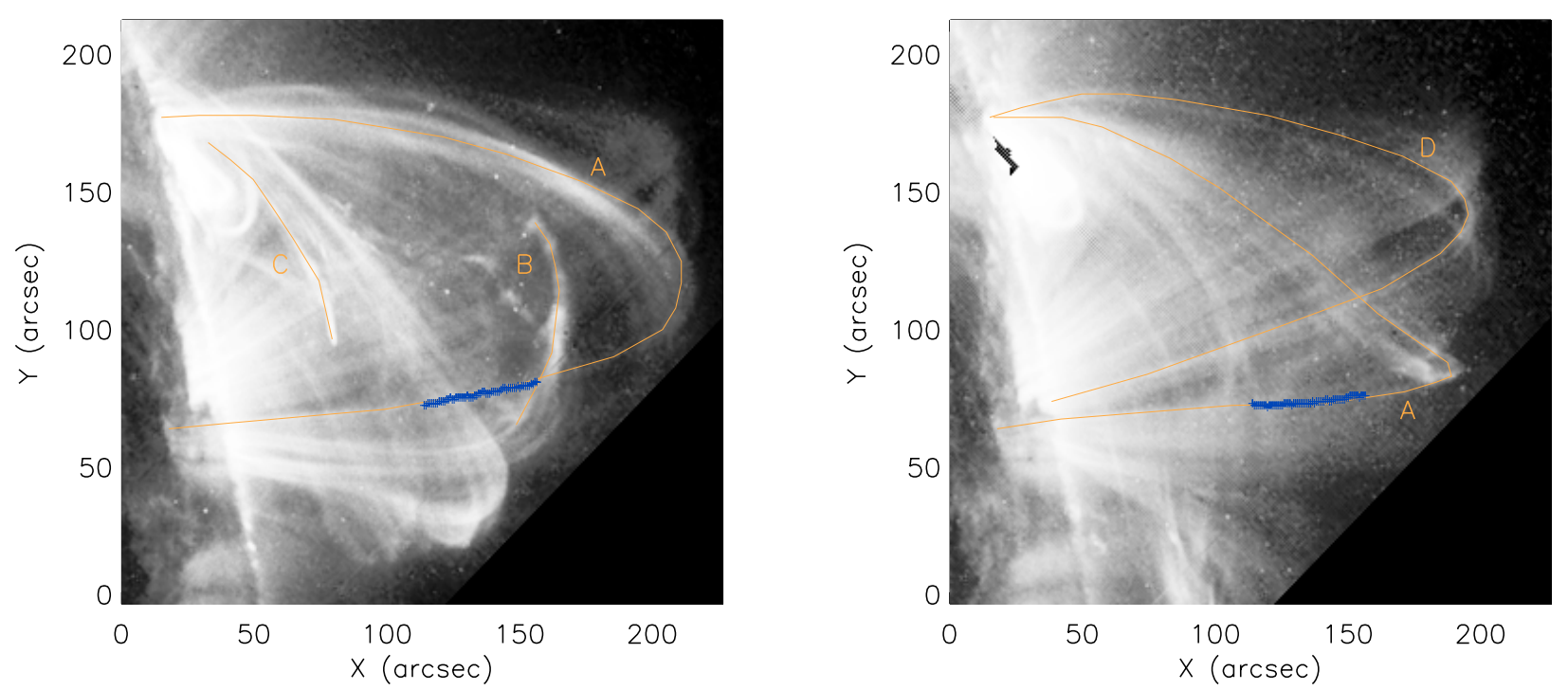

Fig. 1. Left panel: the starting frame of the studied time sequence. A indicates the oscillating loop studied in this paper. B points at the material expelled by the triggering loop (C). Right panel: the 14th frame of the studied time sequence. A indicates the oscillating loop studied in this paper. $\mathrm{D}$ shows another oscillating loop in the same loop complex.

Roberts et al. 1984). Coronal seismology can be achieved by studying oscillations in the corona and comparing the observed properties with models. By adjusting the model, restrictions on physical quantities in the corona can be obtained.

The transverse oscillations are an excellent tool to do coronal seismology with. The coronal dissipative coeffecients were estimated by Nakariakov et al. (1999) and the local magnetic field in the oscillating loop was calculated by Nakariakov \& Ofman (2001). Andries et al. (2005a) used the double periodicity measured by Verwichte et al. (2004) to measure the density scale height in the corona. Verwichte et al. (2006b) determined the radial density structure in oscillation coronal loops, and Arregui et al. (2007) found a lower bound for the internal Alfvén transit time.

Since then, a lot of interest has gone out to multiple periodicities in the same structure. The influence of the density stratification in the corona on the ratio of the period of the fundamental and the second harmonic was studied by McEwan et al. (2006); Dymova \& Ruderman (2006). Unfortunately, the only measurement of such a double periodicity (Verwichte et al. 2004) had very large errors and could not be used to confidently establish the density scale height. More recently, De Moortel \& Brady (2007) observed a loop mainly oscillating as a 2nd harmonic, but also showing a periodicity consistent with the fundamental mode.

In this paper, we report on the high accuracy measurement of a double periodicity in a single loop. The two periods, together with the spatial structure of the oscillation, are used to determine coronal loop parameters which are difficult to measure, such as the density scale height in coronal loops.

\section{Basic properties of the event}

We use the $171 \AA$ observations of the Transition Region And Coronal Explorer (TRACE) satellite (Handy et al. 1999) to study the oscillations in an active region on the $23 \mathrm{rd}$ of November 1998, from 06:35:57 to 06:48:43 UT. The time series has an average cadence time of $33 \mathrm{~s}$.

In the images preceding the studied time series (and in Fig. 1, left), it is observed that a low lying loop is violently disrupted (indicated by $\mathrm{C}$ in Fig. 1, left). After this violent event, a cloud of material is seen to escape the loop system (shown by B in Fig. 1, left), pushing aside the overlying loops (A in Fig. 1, left). Disturbed out of their equilibrium, the overlying loops start to oscillate in the wake of the escaping material. The velocity perpendicular to the line of sight of the expelled material is estimated to be approximately $260 \mathrm{~km} \mathrm{~s}^{-1}$, which is compatible with the values obtained by Wills-Davey \& Thompson (1999); Wills-Davey (2006).

\subsection{Background loop $D$}

The loops (indicated by D in Fig. 1, right) the furthest away from the perturbation have their axis displaced during the oscillation. Because no density oscillations are observed, this suggests that they oscillate in the fundamental kink mode. The indicated loop behaves as a simple damped harmonic oscillator (see Fig. 2) with a period of $425 \mathrm{~s}$ and a damping time of $2300 \mathrm{~s}$. The loop is estimated to be $384 \mathrm{Mm}$ long (the exact procedure is described in the following paragraph).

\subsection{Foreground loop $A$}

Loop A is our main loop of interest. It has also been studied by Aschwanden et al. (2002) (case 3a). They estimated the loop length to be $390 \mathrm{Mm}$.

To estimate the length of the loop, we determine the location of the loop footpoints. Then, we measure the distance between the loop top and the mid point between the loop footpoins. We assume that this distance is the major radius of a semi-circular (toroidal) loop. The length is then $\pi$ times the major radius. This method for the determination of the length does not account for non-circular shape of the loop. Moreover, it is very difficult to determine the exact position of the loop footpoints. It is thus likely that the error on the estimate of the length will be substantial, up to $10 \%$.

By following the above procedure, the length of loop A can be estimated to be initially $440 \mathrm{Mm}$ long. During the 24 frames of the time sequence $(765 \mathrm{~s})$, the loop shortens significantly to $365 \mathrm{Mm}$. The shrinking of the observed loop is of the same 


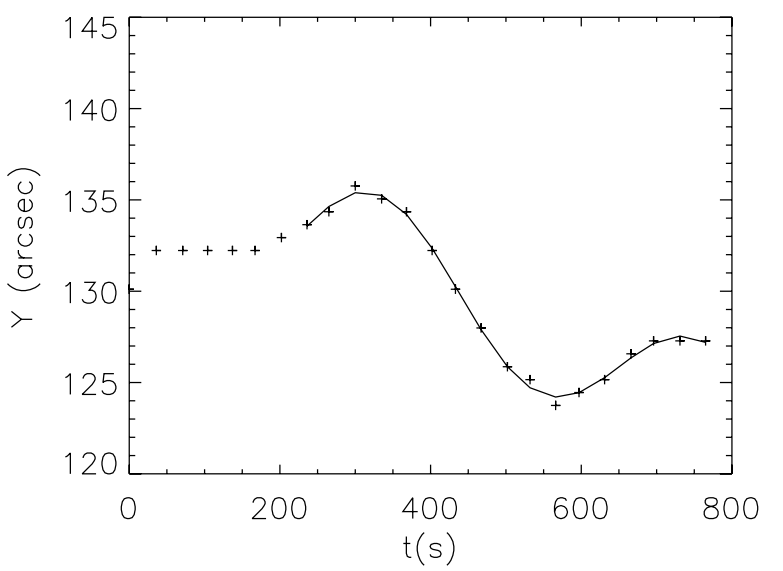

Fig. 2. The vertical displacement of loop D in Fig. 1, right versus time. The actual measurements are indicated with $+\mathrm{s}$, while the fit is indicated with a line. The fitted period is $425 \mathrm{~s}$, the damping time is $2300 \mathrm{~s}$ and the amplitude is 3.95 arcsec.

magnitude as in the report of Li \& Gan (2006). The current loop shrinks $\sim 17 \%$ in $12 \mathrm{~min}$, and the loop in Li \& Gan (2006) shrinks $\sim 30 \%$ in $5 \mathrm{~min}$. On the other hand, Li \& Gan study a short, postflare loop, whereas the loop studied here is very long. The apparent shrinking of the loop may also be caused by a change of the inclination of the loop with respect to the solar surface, or a more complicated change of the loop shape.

These measured lengths are compatible with the values found by Aschwanden et al. (2002).

The loop exhibits a more involved oscillatory pattern than the background loop D. During the oscillation, a "knot" is formed near the loop top (see Fig. 1, right). If the oscillation would be a fundamental mode, the form of the loop would be maintained (Schrijver et al. 2002). The deformation of the loop shape clearly shows that higher harmonics must be involved. On the other hand, the loop axis is still displaced, and no density oscillations are observed. This indicates that the fast kink mode is observed, but that a combination of longitudinal mode numbers is excited. The longitudinal structure of the oscillation suggests that the 2nd harmonic kink oscillation is observed.

The brightening of the loop top by non-linear effects has been studied by Terradas \& Ofman (2004) and is not considered in this paper in detail.

\section{Analysis of the event}

\subsection{Determination of the oscillation characteristics}

To facilitate the analysis, the images are rotated by $45^{\circ}$ in the anti-clockwise direction. By doing this, the loop is almost aligned parallel with the $X$-axis (see Fig. 1). A disadvantage of this rotation is that the resolution reduces by a factor of $\sqrt{2}$, because no interpolation is performed and only pixels with an even sum of indices are retained.

For a fixed horizontal coordinate $(x)$, the vertical position of the loop $(y)$ is estimated throughout the duration of the oscillation. This procedure is repeated for 60 positions along the loop leg. The estimated perturbations $y(x, t)$ are indicated by a blue line in Fig. 1.

To analyse the perturbations, Gaussian noise with a standard deviation of 1.41 arcsec (i.e. 2 pixels in the rotated image) is

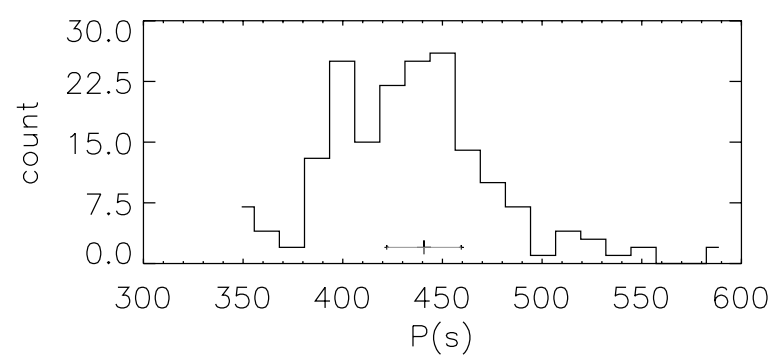

Fig. 3. The distribution of the fitted period to the noisy signal at $x=$ $150.6^{\prime \prime}$. The mean is indicated with a + at the bottom, while the standard deviation is shown by the horizontal error bars on the mean.

added to $y(x, t)$. Then, for a fixed vertical slit (i.e. a fixed $x$ position), the resulting noisy data is fitted with the function

$A \sin \left(\frac{2 \pi t}{P}+\phi\right) \exp (-t / \tau)+C+D t$

where $A$ is the amplitude of the oscillation, $P$ the period, $\phi$ the phase, $\tau$ the damping time and $C$ and $D$ describe the average intercept and global shift of the loop. For each fixed $x$ slit, a set of $\{A, P, \phi, \tau, C, D\}$ is obtained.

The above procedure is repeated 200 times with a varying noise. For each slit, a statistical distribution of parameters is obtained (e.g. Fig. 3 shows the distribution of the best-fitting period for $\left.x=150.6^{\prime \prime}\right)$. From this distribution, the mean and the variance can be computed (both the values are indicated in Fig. 3). We thus obtain the mean values and errors of $\{A, P, \phi, \tau, C, D\}$ for a fixed $x$ value. The fitted signal with these mean parameters is overplotted on the original data in the upper panel of Fig. 4, for $x=150.6^{\prime \prime}$.

Figure 5 shows the dependency of the mean parameters and their errors on $x$. It can be established from Fig. 5 that, for the pixels lying higher up the loop $\left(x \in\left[135.1^{\prime \prime}, 156.3^{\prime \prime}\right]\right)$, a consistent value for the period and phase is obtained. This suggests that the fitting identifies the same oscillation throughout that part of the loop. By averaging over the top part of the loop, a more precise estimate of the oscillation properties can be made. The errors on the oscillation properties are reduced drastically by taking the mean over 31 points. For the considered interval, an average period of $435.6 \pm 4.5 \mathrm{~s}$ is found (see the appendix for details on the error analysis), and an average damping time of $2129 \pm$ $280 \mathrm{~s}$. The value of the period is compatible with the previous estimates for this event (see Aschwanden et al. 2002, case 3a): $P=522 \mathrm{~s}$, the value for the damping time is almost double the previously estimated value: $\tau=1200 \mathrm{~s}$.

As a next step in the analysis, for each $x$ position, the bestfitted function is subtracted from the original signal. As above, the obtained residu and additional Gaussian noise is fitted with Eq. (1). Again, a statistical ensemble is found for each $x$ position. Using the mean parameters, the fitted function is overplotted on the residu in the middle panel of Fig. 4.

The dependence on $x$ of the mean fitting parameters and their errors in the residu signal is shown in Fig. 6. Again a consistent oscillation is detected in the top part of the loop. The average period found in the residu is $242.7 \pm 6.4 \mathrm{~s}$, and the average damping time $872 \pm 221 \mathrm{~s}$.

We conclude that the analysed signal contains two oscillations with different periods, most probably the fundamental mode and the 2nd harmonic. 


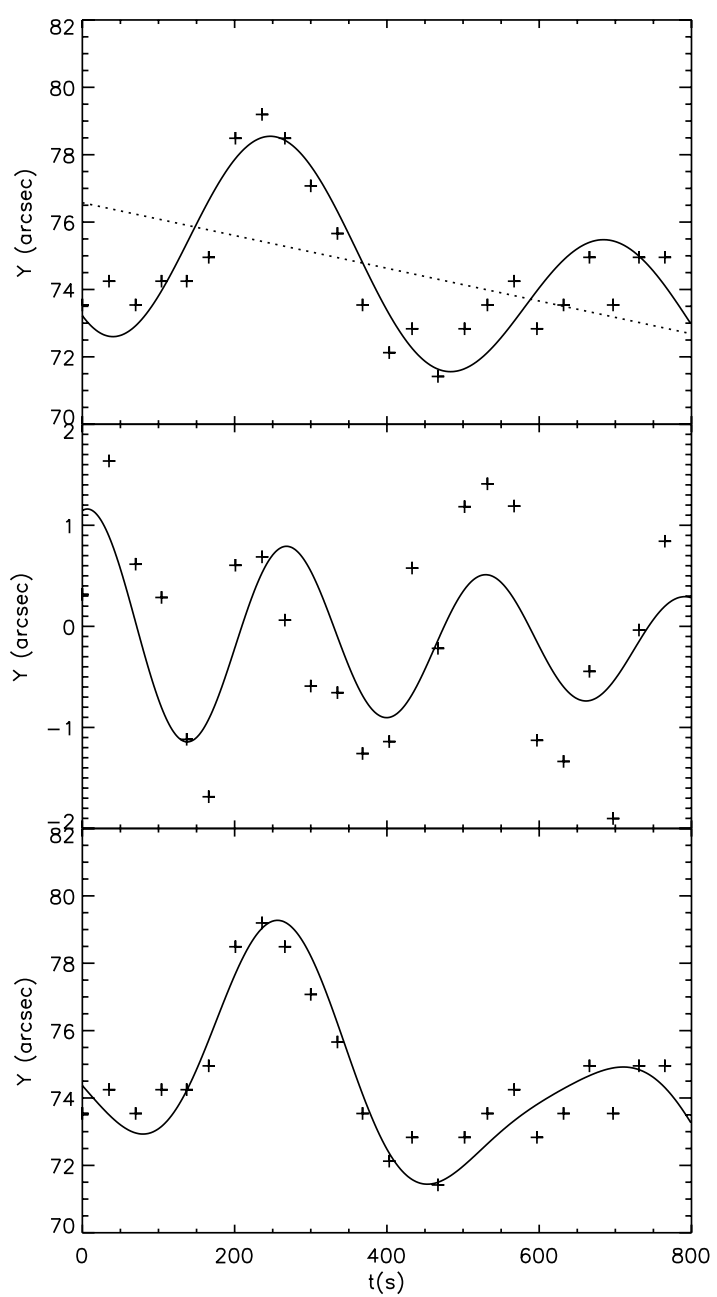

Fig. 4. A typical signal of the transverse displacement of an oscillating loop segment of one-pixel width $(+)$, with the mean fitted signal overplotted (lines) and the trend line (dotted line). The upper panel shows the original signal, while the middle panel shows the fit to the residu. The bottom panel shows the original signal and overplots the combined fits.

\subsection{Coronal loop seismology}

The ratio of periods of these two oscillations can be calculated to be $P_{1} / P_{2}=1.795 \pm 0.051$. This value significantly deviates from 2 , and thus rules out the possibility of a non-linearly excited second harmonics, where the ratio has to be exactly equal to 2 .

According to Nakariakov \& Oraevsky (1995) the appearance of a shorter periodicity in the signal can be connected with the resonant 3-wave interaction of the longer period kink mode with a shorter period kink mode and a sausage mode. In this case, the frequency of the sausage mode would be a sum of the frequencies of interacting kink modes. The sausage mode in the resonant triplet would not be resolved with TRACE because of its short period. However, it is not clear whether the resonant conditions can be satisfied at all in the considered loop. Also, strictly speaking, the theory developed in Nakariakov \& Oraevsky (1995) is only applicable to propagating waves and needs to be extended to describe the interaction between standing modes. Thus, we rule out this interpretation as theoretically undeveloped.

Long-wavelength kink modes of a magnetic cylinder are known to be weakly dispersive (e.g. Nakariakov \& Verwichte 2005). The $P_{1} / P_{2}$ ratio caused by the dispersion in an unstratified long loop is within a few percent of 2 (see Fig. 2

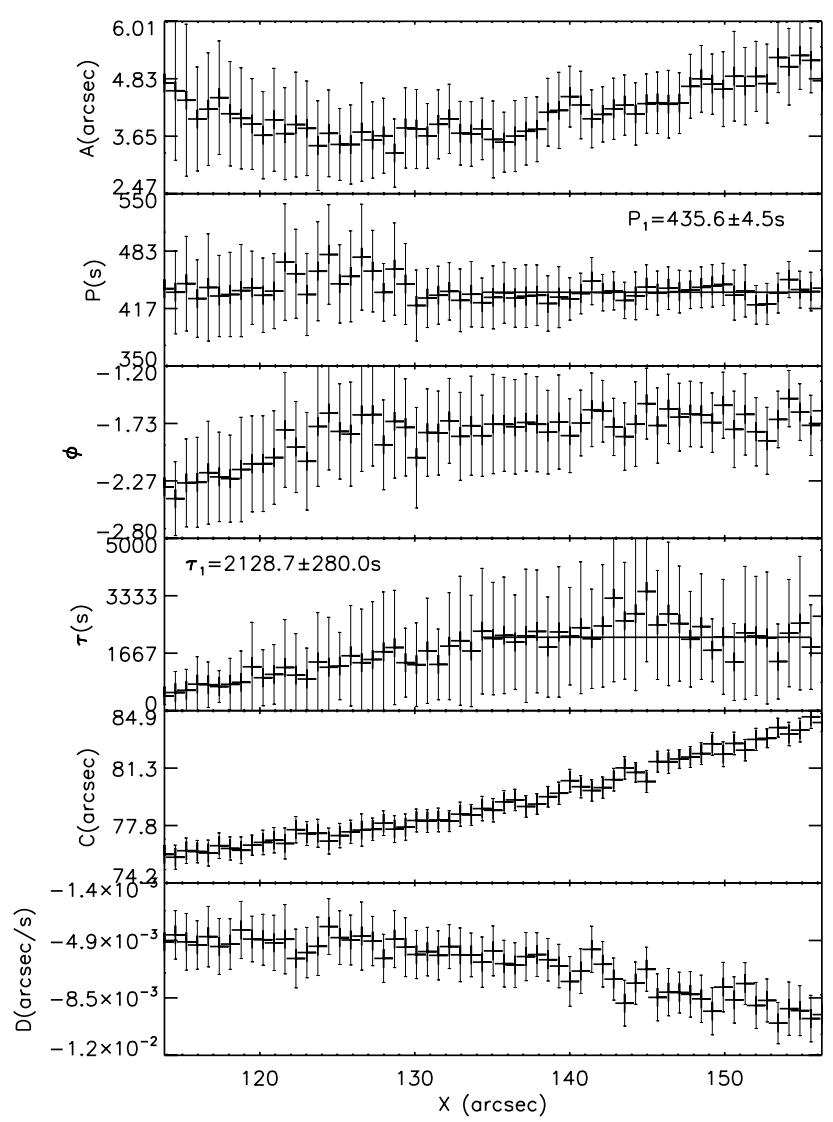

Fig. 5. From top to bottom: $A, P, \phi, \tau, C, D$ for the fit of the original signal of 60 pixels along the loop leg.

in McEwan et al. 2006). The observed deviation of $P_{1} / P_{2}$ from 2 is too large to be explained by dispersion only.

The effect of the vertical density stratification $(\rho(z)=$ $\left.\rho_{0} \exp (-z / H)\right)$ on $P_{1} / P_{2}$ was studied by Andries et al. (2005a); McEwan et al. (2006). Andries et al. assumed a vertical density stratification in the solar corona, and projected this dependency on a cylindrical loop. On the other hand, McEwan et al. took an exponential density stratification in the loop itself. Yet another approach was taken by Dymova \& Ruderman (2006), who did the calculations for non-semi-circular loops in a vertically stratified atmosphere.

If it is assumed that the deviation of $P_{1} / P_{2}$ from 2 is solely caused by the vertical density stratification, a range of values for the relative density stratification $L / \pi H$ can be found ( $L$ is the loop length and $H$ is the density scale height). Using the results of Andries et al. (2005a), we find a value of $L / \pi H=1.17_{-0.3}^{+0.28}$. Similarly, using a slightly different longitudinal density profile, McEwan et al. (2006) would find $L / \pi H=1.08 \pm 0.28$.

In the paper of Dymova \& Ruderman (2006), the height of the geometry centre of the loop $h_{a}$ is allowed to be shifted above $\left(h_{a}<0\right)$ or below the photosphere $\left(h_{a}>0\right)$. Dymova \& Ruderman (2006) calculated the values for $P_{1} / P_{2}$ for different elevations of the loop above the photosphere. Their results are displayed in Fig. 7, the determined density scale height and the errors are also shown.

Using the observed value for $P_{1} / P_{2}$ (without the errorbars), a range of $L / \pi H \in[0.97,1.64]$ is found for a varying $h_{a} \pi / L \in$ $[-0.5,0.5]$ (full line on the horizontal axis). When the errorbars are included in the analysis, a range of $L / \pi H \in[0.73,2.04]$ can be established (dashed line on the horizontal axis). 


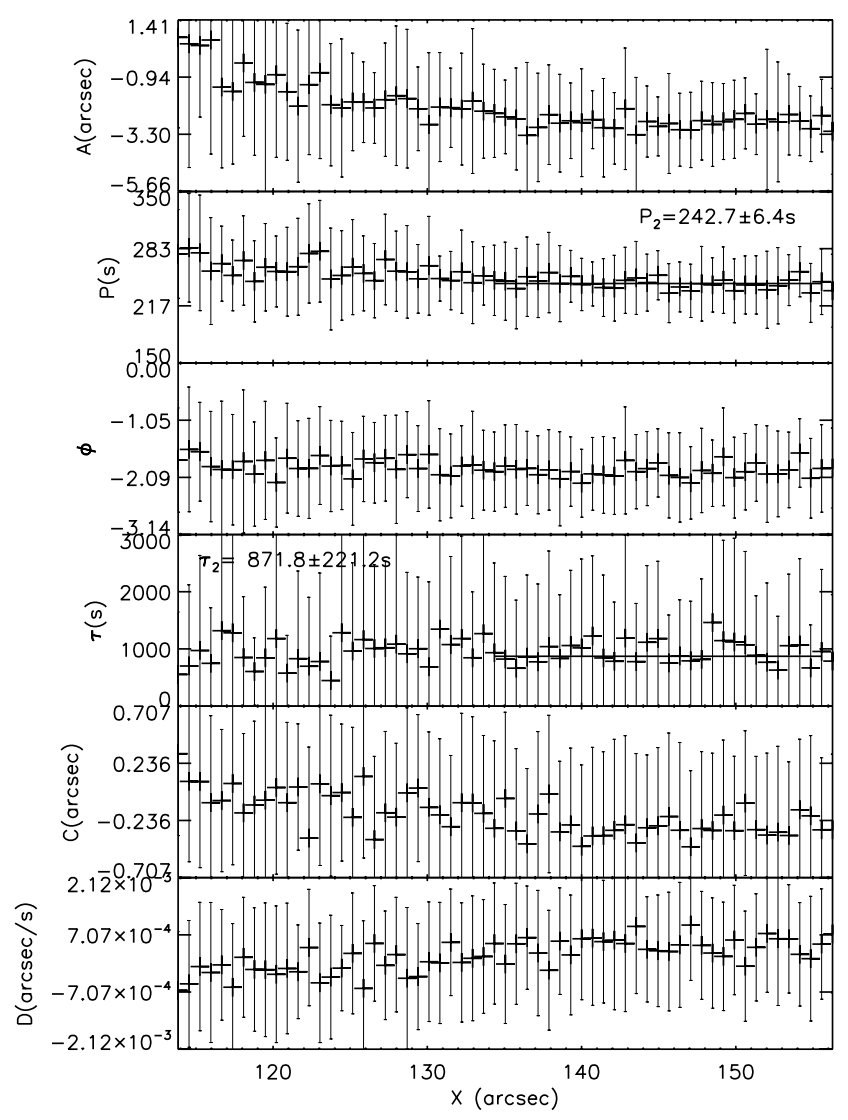

Fig. 6. From top to bottom: $A, P, \phi, \tau, C, D$ for the fit of the residu signal, obtained after subtraction of the fit in Fig. 5 from the original signal.

To estimate the absolute value of the density scale height $H$, an estimate of the length of the loop $L$ is needed. Before the event, the length of the loop is estimated to be $440 \mathrm{Mm}$. At the end of the observations, the loop has shortened to approximately $365 \mathrm{Mm}$. To estimate the density scale height, an average loop length of $400 \mathrm{Mm}$ is assumed.

Using this average length, the estimated value $L / \pi H=$ 1.17 yields a density scale height of $H=109_{-21}^{+37} \mathrm{Mm}$, using the results of Andries et al. (2005a). With the results of McEwan et al. (2006), a density scale height $H=118_{-24}^{+41} \mathrm{Mm}$ is obtained. Taking into account a non-semi-circular geometry, a value $H=109_{-31}^{+22} \mathrm{Mm}$ is established. If the errorbars are included, together with the effects of a non-semi-circular geometry, we find that $H \in[62 \mathrm{Mm}, 174 \mathrm{Mm}]$. These results are summarized in Table 1.

The estimates of the density scale height do not take into account the errors on the loop length. The errors on the loop length may be as large as $10 \%$. This results in an error on the density scale height of up to $10 \%$. For the currently estimated value for $H$, this would be approximately $10 \mathrm{Mm}$. This error is much less than the error induced by the uncertainties on the ratio of the periods.

The seismologically estimated value for $H=109 \mathrm{Mm}$ is more than double the value of $50 \mathrm{Mm}$, expected in a hydrostatically stratified plasma with a temperature of $1 \mathrm{MK}$, corresponding to the observational bandpass $171 \AA$. This is not abnormal, as the stratification inside coronal loops may exceed the hydrostatical value by a factor of 4 (see Aschwanden et al. 2000, 2001). Even stronger, Aschwanden et al. (2000) show in their Fig. 7 that the ratio of the density scale heights in coronal loops and the

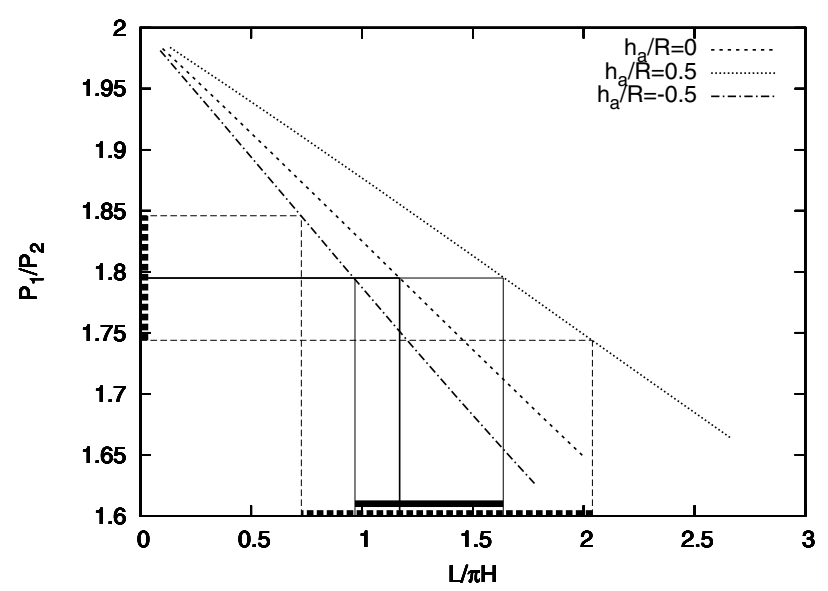

Fig. 7. The results from Dymova \& Ruderman (2006) plotted with a dashed line (semi-circular loop, identical to Andries et al. 2005a), dotted line (shortened loop with centre $L / \pi 2$ below the photosphere), dashdotted line (longer loop with centre $L / \pi 2$ above the photosphere). The interval for $P_{1} / P_{2}$ introduced by the error bars is indicated on the vertical axis with a fat dashed line. On the horizontal axis, the interval for $L / \pi H$ introduced by the non-semi-circularity is shown by a fat full line. The range for $L / \pi H$ obtained by using both the errors and the non-semicircularity is shown by a fat dashed line.

hydrostatic scale height exhibits an increasing trend for longer loops.

Our estimate may point out that coronal loops have a higher density scale height inside the loop when compared to the surrounding corona.

\subsection{Tests of damping mechanisms}

In the previous subsection, we only considered the ratio $P_{1} / P_{2}$ and did not take into account the ratio of the damping times. The ratio $\tau_{1} / \tau_{2}=2.55 \pm 0.73$. This ratio is significantly higher than $P_{1} / P_{2}$. This could point out that the damping mechanism in coronal loops is dependent on the wave number.

For a more rigorous approach, we assume that the damping time goes as $C k^{n}$, where $C$ is a constant solely depending on the equilibrium parameters of the loop and $k$ is the longitudinal wave number. We can then calculate that

$2.55 \pm 0.73=\frac{\tau_{1}}{\tau_{2}}=\left(\frac{k_{1}}{k_{2}}\right)^{n}=\left(\frac{1}{2}\right)^{n}$

and thus deduce that $n=-1.35_{-0.36}^{+0.49}$. This interval for $n$ accomodates the value $n=-1$.

Alternatively, the damping time can be rescaled to the period: $\tau \sim P^{p}$. Since the scaling factor can be assumed to only depend on loop parameters, it can be established that

$\frac{\tau_{1}}{\tau_{2}}=\left(\frac{P_{1}}{P_{2}}\right)^{p}$, and thus that $p=\frac{\log \left(\frac{\tau_{1}}{\tau_{2}}\right)}{\log \left(\frac{P_{1}}{P_{2}}\right)}$.

Using the observed values, we can estimate that $p=1.60$ and, taking the errors into account, has to be between 0.98 and 2.14.

These values and confidence intervals for $n$ and $p$ provide a restriction on any proposed damping mechanism.

For resonant absorption, $n=-1$ and $p=1$. This means that resonant absorption can explain the currently observed damping times. 
Table 1. A summary of the density scale heights in the oscillating loop, estimated using different methods. Models (1), (2) and (3) are taken from Andries et al. (2005a); McEwan et al. (2006); Dymova \& Ruderman (2006) respectivily.

\begin{tabular}{cccc}
\hline \hline Physical parameter & Model (1) & Model (2) & Model (3) \\
\hline$H(\mathrm{Mm})$ & $109_{-21}^{+37}$ & $118_{-24}^{+41}$ & $109_{-31}^{+22}$ \\
& & & (due to non-semi-circularity) \\
\hline
\end{tabular}

For lateral wave leakage, $p$ is expected to be negative (Verwichte et al. 2006a), i.e. for higher harmonics, the radial extent in smaller, less leakage is present and the damping time will be longer. The observationally established interval for $p$ does not accomodate negative values. This means that, in this case, the damping cannot be explained by lateral wave leakage.

\subsection{Amplitude dependence on the vertical coordinate}

For a stratified medium, the fundamental mode is essentially coupled to the 3rd harmonic (see Andries et al. 2005b) without assuming a damping mechanism. The eigenfunction will thus be of the form

$A(\sin (\theta)+a \sin (3 \theta))$,

where $A$ is the global amplitude of the fundamental harmonic, $\theta$ is the arc length along the loop $(\theta \in[0, \pi])$ and the coupling parameter $a$ depends of the importance of the density stratification, measured by $L / \pi H$. Ignoring the coupling effect on the second harmonics, we expect the eigenfunction of the overtone to be

$B \sin (2 \theta)$,

where $B$ is the amplitude of the second harmonics, assumed to be independent of $A$ and solely determined by the form of the original perturbation.

If it is assumed that the line of sight is parallel to the loop baseline, the vertical height above the Sun $z$ can be written as:

$z=L / \pi \sin \theta, \quad$ leading to $\quad \theta=\arcsin \pi z / L$.

Substituting this formula in Eqs. (2)-(3), we find the expected amplitude dependence on the vertical coordinate. For the fundamental mode, we obtain

$A\left((1+3 a) \frac{\pi z}{L}-4 a\left(\frac{\pi z}{L}\right)^{3}\right)$,

and for the 2 nd harmonics, we find

$2 B \frac{\pi z}{L} \sqrt{1-\left(\frac{\pi z}{L}\right)^{2}}$

However, when fitting the function for the fundamental mode to the top panel of Fig. 5, and the function for the 2nd harmonics to the top panel of Fig. 6, no conclusive results are obtained. We find $A=8.9 \pm 3.5 \mathrm{px}, a=0.02 \pm 0.36, B=-4.1 \pm 0.5 \mathrm{px}$, $\pi z_{0} / L=0.81 \pm 0.25, \pi z_{1} / L=0.56 \pm 0.25$, where $z_{0}$ and $z_{1}$ indicate the position of the highest and lowest measured vertical slit, respectively. The value of $a$ yields a range for $L / \pi H \in[0,3.7]$ and thus reveals no extra information.

We know that the distance between $z_{0}$ and $z_{1}$ is exactly $30 \mathrm{px}=15.3 \mathrm{Mm}$. We can thus calculate that $L \approx 192 \mathrm{Mm}$, in a range of $L \in[79 \mathrm{Mm}, \infty[$. The observationally estimated value for the length $(\approx 400 \mathrm{Mm})$ is higher than this seismological estimate, but lies within the exorbitant errorbars.

Using a more simple approach and neglecting all coupling and higher harmonics (i.e. take $B=0$ and $a=0$ ), the top panel of Fig. 5 can be fitted with a straight line. From this fit, it is found that the amplitude will be 0 for $x=82.7$ arcsec. However, from the observations, it can be estimated that the footpoint is situated approximately at $x=17.7$ arcsec. These two values do not agree at all.

Of course, this analysis did not include the effect of a noncircular loop shape. In that case, it can be expected that a fundamental mode can still be described as

$A \sin (\theta)+$ correction terms,

and that the correction terms will be smaller than the fundamental mode. However, the correction terms may include a contribution from the 2nd harmonic (due to the geometry), which could alter the analysis.

\subsection{Alfvén speeds in the loop complex}

If the measured periods in loops $\mathrm{A}$ and $\mathrm{D}$ belong to the fundamental kink mode, the kink phase speed can be estimated in these loops. For loop A, we find a value $C_{\mathrm{k}}=2 L / P=$ $1800 \mathrm{~km} \mathrm{~s}^{-1}$. Loop D is slightly shorter, and has a slightly shorter period, so that the kink phase speed is estimated to have the same value as in loop A.

The number density is estimated to be $4.6 \times 10^{14} \mathrm{~m}^{-3}$ and $6.8 \times 10^{14} \mathrm{~m}^{-3}$ for, respectively, loop A and D. To measure the values, we assumed that the emission in the loop was generated in a layer of thickness $2 R$ (the loop radius $R$ is measured by fitting the loop emission with a Gaussian) by a plasma with a fixed temperature of $0.95 \times 10^{6} \mathrm{~K}$, i.e. the peak temperature of the TRACE $171 \AA$ filter. If the temperature of the emitting volume would be different, the plasma density would increase. As such, these estimated densities are a lower limit to the actual density. The density is then calculated by $\rho_{\mathrm{A}}=\sqrt{\left(I_{\mathrm{A}}-I_{\mathrm{e}}\right) / 2 \chi R}$, where $I_{\mathrm{A}}$ and $I_{\mathrm{e}}$ are the intensities in loop A and in the exterior plasma, respectively, and $\chi$ is the TRACE $171 \AA$ response function (taken from SolarSoft). The values for the density were not corrected for line of sight integration and are calculated in the assumption that the emitting loop is perpendicular to the line of sight.

Taking into account that the measured phase speeds $C_{\mathrm{kA}}$ and $C_{\mathrm{kD}}$ and lengths of loops $\mathrm{A}$ and $\mathrm{D}$ are approximately equal to each other, we now can write down that:

$1=\frac{C_{\mathrm{kA}}}{C_{\mathrm{kD}}}=\frac{B_{\mathrm{A}}}{B_{\mathrm{D}}} \sqrt{\frac{\rho_{\mathrm{e}}+\rho_{\mathrm{D}}}{\rho_{\mathrm{e}}+\rho_{\mathrm{A}}}} \approx \frac{B_{\mathrm{A}}}{B_{\mathrm{D}}} \sqrt{\frac{n_{\mathrm{D}}}{n_{\mathrm{A}}}}=1.2 \frac{B_{\mathrm{A}}}{B_{\mathrm{D}}}$,

where quantities with subscript $\mathrm{A}$ and $\mathrm{D}$ belong to the respective loop, $\rho_{\mathrm{e}}$ is the density outside the loops and is assumed to be small with respect to the loop densities. This assumption is not strictly valid for oscillating coronal loops (the internal density is between 1 and 5 times the external density, see Aschwanden et al. 2003). In our case the value 1.2 is an upper limit to $\sqrt{\left(\rho_{\mathrm{e}}+\rho_{\mathrm{D}}\right) /\left(\rho_{\mathrm{e}}+\rho_{\mathrm{A}}\right)}$ and the lower limit would be 1.1 in the case of $\rho_{\mathrm{e}}=\rho_{\mathrm{D}}$.

Equation (4) can be used to estimate the ratio of the magnitude of the magnetic field in the two coronal loops to be $B_{\mathrm{D}} / B_{\mathrm{A}} \approx 1.2$. This suggests that, for loops with a similar length, 
Table 2. A summary of periodicities, lengths and density scale heights for the events mentioned in this article. References. (1) Path C and D are measured by Verwichte et al. (2004).

\begin{tabular}{rcccccc}
\hline \hline & $P_{1}(\mathrm{~s})$ & $P_{2}(\mathrm{~s})$ & $P_{1} / P_{2}$ & $L / \pi H$ & $L(\mathrm{Mm})$ & $H(\mathrm{Mm})$ \\
\hline Current event & $435.6 \pm 4.5$ & $242.7 \pm 6.4$ & $1.795 \pm 0.051$ & $1.17_{-0.30}^{+0.28}$ & 400 & $109_{-21}^{+37}$ \\
path C (1) & $447.7 \pm 15.8$ & $246.5 \pm 6.0$ & $1.82 \pm 0.08$ & $1.02_{-0.44}^{+0.46}$ & 218 & $68_{-21}^{+52}$ \\
path D (1) & $387.4 \pm 7.5$ & $244.8 \pm 7.7$ & $1.58 \pm 0.06$ & $2.43_{-0.36}^{+0.38}$ & 228 & $30_{-4}^{+5}$ \\
\hline
\end{tabular}

the magnetic field has roughly the same magnitude throughout different loops in the same active region.

Considering the absolute value of the magnetic field, we can state that:

$10 \times 10^{-4} \mathrm{~T}=\sqrt{\frac{\mu \rho_{A} C_{\mathrm{k}}^{2}}{2}} \leq B_{\mathrm{A}} \leq \sqrt{\mu \rho_{\mathrm{A}} C_{\mathrm{k}}^{2}}=14 \times 10^{-4} \mathrm{~T}$,

where the two outer limits are obtained in the case of a loop embedded in vacuum and a loop with density equal to the external density, respectively. These values are compatible with the value obtained by Nakariakov \& Ofman (2001); Verwichte et al. (2004). The outer limits obtained above do not take into account the errors on the density measurement, and merely indicate the errors introduced by the unknown density contrast between the internal of the loop and the external plasma.

The results in this subsection have to be taken with caution: the errors on both the density and, to a lesser extent, the length are large. As such, the values found in this subsection are only an indication of the order of magnitude of the physical quantities.

\section{Verwichte et al. (2004) revisited}

In the currently studied event, the measurement errors on the periods were drastically reduced by averaging the period over a whole segment of the oscillating loop. A loop segment was also studied by Verwichte et al. (2004), but a different averaging formula was used, based on the standard deviation of the data cloud rather than the averaging of the measurement errors. However, when redoing the analysis and using Eqs. (A.1)-(A.2), a more precise determination of the periods is obtained.

Their path $\mathrm{C}$ now has $P_{1}=447.7 \pm 15.8 \mathrm{~s}$ and $P_{2}=246.5 \pm$ $6.0 \mathrm{~s}$, leading to $P_{1} / P_{2}=1.82 \pm 0.08$. Using the model of Andries et al. (2005a), we find $L / \pi H=1.02_{-0.44}^{+0.46}$. Using the length $L=218 \mathrm{Mm}$ of that loop, a density scale height $H=$ $68_{-21}^{+52} \mathrm{Mm}$ is obtained.

Similarly, their path D shows periodicities $P_{1}=387.4 \pm$ $7.5 \mathrm{~s}$ and $P_{2}=244.8 \pm 7.7 \mathrm{~s}$, resulting in $P_{1} / P_{2}=1.58 \pm 0.06$ and $L / \pi H=2.43_{-0.36}^{+0.38}$. Together with the estimated length $L=$ $228 \mathrm{Mm}$, we find a density scale height $H=30_{-4}^{+5} \mathrm{Mm}$.

These values for the density scale height differ strongly from the value we obtain in the currently studied event. This fact, however, is compatible with Fig. 7 in Aschwanden et al. (2000). That graph shows that the scale height in shorter coronal loops (as is the case in Verwichte et al. 2004) is expected to be lower than for longer coronal loops (current event). Also, the loops studied by Verwichte et al. (2004) are post-flare loops and may not have settled into equilibrium.

A summary of all the periods, lengths and density scale heights of the events studied in Verwichte et al. (2004) and the current paper is given in Table 2 .

\section{Conclusions}

In this article, TRACE $171 \AA$ observations of an active region were analysed. By tracing out a loop segment, two oscillation periods could be detected with high confidence: $435.6 \pm 4.5 \mathrm{~s}$ and $242.7 \pm 6.4 \mathrm{~s}$. Using these periods, and interpreting them as the fundamental and the 2 nd harmonic oscillation, a value $P_{1} / P_{2}=1.795 \pm 0.051$ was found.

This value allowed us to establish $L / \pi H=1.17$, and lead to a density scale height of $H=109_{-21}^{+37} \mathrm{Mm}$. Such a value for the density scale height in the loop is significantly higher than the hydrostatically expected value $(50 \mathrm{Mm})$. Our result thus suggests that the density scale height in coronal loops is much higher than that in the surrounding corona, a result also found by Aschwanden et al. (2000).

We used the measurements for the damping times to study the viability of different damping mechanisms. We were able to find that the damping time must be approximately inversely proportional to the wave number. By relating the ratio of damping times and periods, we found that $\tau \sim P^{1.60}$. Using the relations between the damping time, the wave number and the period, we have established that the observed damping times can be explained by resonant absorption and not by lateral wave leakage.

Furthermore, we estimated that the magnetic field in the observed loop is between 10 and $14 \mathrm{G}$ and thus in the same range as Nakariakov \& Ofman (2001). By comparing the observed oscillation with an oscillation in a neighbouring loop in the same active region, we were able to establish that the ratio of the magnetic fields is between 1.1 and 1.2. This suggests that, for loops with a similar length, the magnetic field remains almost constant throughout an active region.

We used the eigenfunction to obtain geometrical properties of the loop, but found that the observational errors are to large to achieve this. A loop length $L=192 \mathrm{Mm}$ in a range of [75 Mm, $\infty$ [ was estimated. This value of $L$ is not close to the observed loop length, but lies within the errorbars.

Lastly, we revisited the results of Verwichte et al. (2004) and used a different statistical method to reduce the errors on the measurements of the periods. For those events, density scale heights of $H=68_{-21}^{+52} \mathrm{Mm}$ and $H=30_{-4}^{+5} \mathrm{Mm}$ are thus found. These values differ significantly from the value found in our event. This fact, however, is compatible with the findings of Aschwanden et al. (2000).

Acknowledgements. T.V.D. would like to acknowledge the financial support of PPARC.

\section{Appendix A: Estimates of errors}

To calculate the errors on the mean periods, it was assumed that all fitted estimates of the period $P^{i}=P(x=i)$ along the loop were taken from a Gaussian distribution with a variance of $\left(\sigma^{i}\right)^{2}$. Statistical analysis states that the mean period $\bar{P}$ is calculated as:

$\bar{P}=\frac{\sum_{i=0}^{N-1} P^{i} /\left(\sigma^{i}\right)^{2}}{\sum_{i=0}^{N-1} 1 /\left(\sigma^{i}\right)^{2}}$ 
The error on the mean can be calculated by:

$\sigma_{\bar{P}}^{2}=\frac{1}{\sum_{i=0}^{N-1} 1 /\left(\sigma^{i}\right)^{2}}$.

The errors of $P_{1} / P_{2}$ can be calculated by:

$\left(\frac{\sigma_{P_{1} / P_{2}}}{P_{1} / P_{2}}\right)^{2}=\left(\frac{\sigma_{P_{1}}}{P_{1}}\right)^{2}+\left(\frac{\sigma_{P_{2}}}{P_{2}}\right)^{2}$,

if it is assumed that the errors on $P_{1}$ and $P_{2}$ are uncorrelated.

\section{References}

Andries, J., Arregui, I., \& Goossens, M. 2005a, ApJ, 624, L57

Andries, J., Goossens, M., Hollweg, J. V., Arregui, I., \& Van Doorsselaere, T. 2005b, A\&A, 430, 1109

Arregui, I., Andries, J., van Doorsselaere, T., Goossens, M., \& Poedts, S. 2007, A\&A, 463, 333

Aschwanden, M. J., Fletcher, L., Schrijver, C. J., \& Alexander, D. 1999, ApJ, 520,880

Aschwanden, M. J., Nightingale, R. W., \& Alexander, D. 2000, ApJ, 541, 1059

Aschwanden, M. J., Schrijver, C. J., \& Alexander, D. 2001, ApJ, 550, 1036

Aschwanden, M. J., De Pontieu, B., Schrijver, C. J., \& Title, A. M. 2002, Sol. Phys., 206, 99

Aschwanden, M. J., Nightingale, R. W., Andries, J., Goossens, M., \& Van Doorsselaere, T. 2003, ApJ, 598, 1375

De Moortel, I., \& Brady, C. S. 2007, ApJ, Accepted

Dymova, M. V., \& Ruderman, M. S. 2006, A\&A, 459, 241

Edwin, P. M., \& Roberts, B. 1983, Sol. Phys., 88, 179
Goossens, M., Ruderman, M. S., \& Hollweg, J. V. 1995, Sol. Phys., 157, 75 Goossens, M., Andries, J., \& Aschwanden, M. J. 2002, A\&A, 394, L39 Handy, B. N., Acton, L. W., Kankelborg, C. C., et al. 1999, Sol. Phys., 187, 229 Li, Y. P., \& Gan, W. Q. 2006, ApJ, 644, L97

Lin, H., Penn, M. J., \& Tomczyk, S. 2000, ApJ, 541, L83

Lin, H., Kuhn, J. R., \& Coulter, R. 2004, ApJ, 613, L177

McEwan, M. P., Donnelly, G. R., Díaz, A. J., \& Roberts, B. 2006, A\&A, 460, 893

Nakariakov, V. M., \& Ofman, L. 2001, A\&A, 372, L53

Nakariakov, V. M., \& Oraevsky, V. N. 1995, Sol. Phys., 160, 289

Nakariakov, V. M., \& Verwichte, E. 2005, Living Rev. Sol. Phys., 2, 3

Nakariakov, V. M., Ofman, L., DeLuca, E. E., Roberts, B., \& Davila, J. M. 1999, Science, 285, 862

Ofman, L., \& Aschwanden, M. J. 2002, ApJ, 576, L153

Roberts, B., Edwin, P. M., \& Benz, A. O. 1984, ApJ, 279, 857

Ruderman, M. S., \& Roberts, B. 2002, ApJ, 577, 475

Schrijver, C. J., Aschwanden, M. J., \& Title, A. M. 2002, Sol. Phys., 206, 69

Terradas, J., \& Ofman, L. 2004, ApJ, 610, 523

Terradas, J., Oliver, R., \& Ballester, J. L. 2006, ApJ, 642, 533

Uchida, Y. 1970, PASJ, 22, 341

Van Doorsselaere, T., Andries, J., Poedts, S., \& Goossens, M. 2004a, ApJ, 606, 1223

Van Doorsselaere, T., Debosscher, A., Andries, J., \& Poedts, S. 2004b, A\&A, 424,1065

Verwichte, E., Nakariakov, V. M., Ofman, L., \& Deluca, E. E. 2004, Sol. Phys., 223, 77

Verwichte, E., Foullon, C., \& Nakariakov, V. M. 2006a, A\&A, 449, 769

Verwichte, E., Foullon, C., \& Nakariakov, V. M. 2006b, A\&A, 452, 615

Wang, T. J., \& Solanki, S. K. 2004, A\&A, 421, L33

Wills-Davey, M. J. 2006, ApJ, 645, 757

Wills-Davey, M. J., \& Thompson, B. J. 1999, Sol. Phys., 190, 467

Zaitsev, V. V., \& Stepanov, A. V. 1975, Issled. Geomagn. Aeron. Fiz. Solntsa, 3 